\title{
Coexisting attractors, chaos control and synchronization in a self-exciting homopolar dynamo system
}

\author{
Xingrong Chen ${ }^{1}$, Li Xiao ${ }^{1}$, Sifeu Takougang Kingni ${ }^{2 *}$, Irene Moroz ${ }^{3}$, Zhouchao Wei ${ }^{1}$, \\ Hadi Jahanshahi ${ }^{4}$ \\ ${ }^{1}$ School of Mathematics and Physics, China University of Geosciences, Wuhan 430074, China \\ ${ }^{2}$ Department of Mechanical and Electrical Engineering, Faculty of Mines and Petroleum \\ Industries, University of Maroua, P.O. Box 46, Maroua, Cameroon \\ ${ }^{3}$ Mathematical Institute, University of Oxford, Oxford, OX2 6GG, England \\ ${ }^{4}$ Department of Aerospace Engineering, University of Tehran, Tehran, 14395-1561, Iran
}

\begin{abstract}
Purpose: The purpose of this paper is to investigate coexisting attractors, chaos control and synchronization in a self-exciting homopolar dynamo system in this paper.

Design/methodology/approach: Two single controllers are designed and added to the proposed 3D autonomous chaotic system and its stability at zero equilibrium point is guaranteed by applying an appropriate control signal based on Lyapunov stability theory.

Findings: Numerical simulations reveal that the proposed 3D dynamo system exhibits periodic oscillations, double-scroll chaotic attractors and coexisting attractors. Finally, a single controller is designed for the global asymptotic synchronization of unidirectional coupled identical 3D autonomous chaotic system.

Originality/value: The derived results of this paper are new and complement some earlier works. Innovation of this paper concludes two points: coexisting attractors are found and an appropriate control signal based on Lyapunov stability theory is established. The ideas of this paper can be applied to investigate some other homopolar dynamo systems.
\end{abstract}

Keywords: Chaos, Coexisting attractors, Chaos control, Synchronization.

Paper type: Research paper

\section{Introduction}

The study of chaotic systems has been an important and yet not easy task for nonlinear dynamics. Chaotic attractors can be considered as some useful tools to design complex hyperchaotic attractors and communication encryption [1-5]. Based on the analysis of Hilbert's 16th problem on the number and location of limit cycles [6], hidden oscillations can 
be distinguished as a feature in and electrical circuits and electromechanical rotational models $[7]$.

From the aspect that the main geomagnetic field produced by self-exciting magnetohydrodynamic dynamo action in the Earth's liquid metallic core and the 'El NinoSouthern Oscillation' of the Earth's atmosphere-ocean system, three coupled nonlinear ordinary differential equations have been given to describe the behaviour of a self-exciting Faraday disk homopolar dynamo [8-12], which has been the focus of attention for a series of recent studies. The equations for self-exciting homopolar dynamos can be used as the basis of nonlinear low-dimensional analogues in the study of the temporal behaviour of certain phenomena of interest in geophysical fluid dynamics. The proposed disc dynamo has the following form [8]

$$
\begin{array}{ll}
\frac{d x}{d t}=x(y-1)-\beta z, & \\
\frac{d y}{d t}=\alpha\left(1-x^{2}\right)-k y, \\
\frac{d y}{d t}=x-\lambda z,
\end{array}
$$

where $\alpha, \beta, k, \lambda$ are parameters and $x, y, z$ are state variables. The behavior of the system (1), including its sensitivity to initial conditions, depends on the four parameters ( $\alpha, \beta, k, \lambda)$, the least interesting case being when the system fails to function as a self-exciting dynamo capable of amplifying a small adventitious electric current. However, the further results should be needed to elucidate more rich and complex behaviour indicated in disk homopolar dynamos.

In recent years, more researchers have paid attention to multistability [13-16]. In particular, as a rich character in many non-linear problems. hidden attractors have not been studied thoroughly in many realistic chaotic systems, such as some deep-seated complex behaviours [17-24]. The proposed disc dynamo (1) means the magnetic field inside the liquid part of the Earth's core will be much more complex than that at the surface. In 2015, Wei et al. have considered an extended Rikitake system, and found the bifurcations and hidden chaos [25]. In 2016, from the viewpoint of mathematics a four disk dynamos model has been presented and considered by eight degrees of freedom [26]. Recently, some hidden attractors both can been found in the 3D, 4D and 5D self-exciting homopolar disc dynamos [27-30]. However, many important questions, such as complex chaotic behaviour and the effect of multiple time delays, are still not solved theoretically. In 2019, the impact of multiple time delays on the dynamics of two-disc dynamos is studied and research results reveal a classical period- 
doubling route towards deterministic chaos in disc dynamo [31]. Therefore, the effect of multistability is considered as an important factor, which will be closer to reality. Compared to the case of single time delay or the case without delay, research on multistability will be closer to the actuality and helpful to understand the disc dynamos.

Our objective is to investigate coexisting attractors, chaos control and synchronization in a self-exciting homopolar dynamo system in the paper. Here, the frame of this article is constructed: In Section 2, some basic analysis of homopolar dynamo system is considered. Bifurcation diagrams, Lyapunov exponents, phase portraits and cross section of the basin of attraction are considered for multistability. In Section 3, two single controllers are mathematically designed by using the principle of Lyapunov's method for asymptotic global stability. In Section 4, a single feedback control method is used to synchronize unidirectional coupled identical 3D chaotic systems. Finally, the conclusions are stated in Section 5

\section{Dynamical analysis of 3D chaotic system}

When multiplied value of mechanical friction and self-inductance is equal to the multiplicative value between total series resistance and inertia in the disk ( $k=1$ in Ref.[6]), the self-exciting single-disk homopolar dynamos system is given as in below:

$$
\begin{aligned}
& \frac{d x}{d t}=x(y-1)-\beta z, \\
& \frac{d y}{d t}=\alpha\left(1-x^{2}\right)-y, \\
& \frac{d y}{d t}=x-\lambda z .
\end{aligned}
$$

System (2) has a symmetry under the following transformation: $S(x, y, z) \rightarrow(-x, y,-z)$ For $1-\frac{1}{\alpha}\left(1+\frac{\beta}{\lambda}\right)<0$, system (2) has only one equilibrium point $E_{0}(0, \alpha, 0)$. While for $\alpha \neq 0$, $\lambda \neq 0$ and $1-\frac{1}{\alpha}\left(1+\frac{\beta}{\lambda}\right) \geq 0$, it has three equilibrium points $E_{0}(0, \alpha, 0)$ and $E_{1,2}\left( \pm x_{0}, \alpha\left(1 \mp x_{0}\right), \pm \frac{x_{0}}{\lambda}\right)$ with $x_{0}=\sqrt{1-\frac{1}{\alpha}\left(1+\frac{\beta}{\lambda}\right)}$. The characteristic equation of system

evaluated at the equilibrium point $E_{0}$ is:

$$
\Lambda^{3}+(2+\lambda-\alpha) \Lambda^{2}+(1+2 \lambda+\beta-\alpha-\lambda \alpha) \Lambda+\beta+\lambda(1-\alpha)=0 \text {. }
$$

Using the Routh-Hurwitz conditions, Eq. (3) has all roots with negative real parts if and only if 


$$
2+\lambda-\alpha>0, \beta+\lambda(1-\alpha)>0,(2+\lambda-\alpha)(1+2 \lambda+\beta-\alpha-\lambda \alpha)-[\beta+\lambda(1-\alpha)]>0 .
$$

The characteristic equation of system (2) evaluated at the equilibrium point $E_{1}$ is:

$$
\begin{aligned}
& \Lambda^{3}+\left(2+\lambda+\alpha x_{0}-\alpha\right) \Lambda^{2}+\left(1+2 \lambda+\beta+\alpha x_{0}+\alpha \lambda x_{0}+2 \alpha x_{0}^{2}-\alpha-\lambda \alpha\right) \Lambda+\beta+\lambda \\
& \quad+\alpha \lambda\left(2 x_{0}^{2}+x_{0}-1\right)=0 .
\end{aligned}
$$

Applying the Routh-Hurwitz conditions, Eq. (5) has all roots with negative real parts if and only if

$$
\begin{gathered}
2+\lambda+\alpha x_{0}-\alpha>0 \beta+\lambda+\alpha \lambda\left(2 x_{0}^{2}+x_{0}-1\right)>0 \\
\left(2+\lambda+\alpha x_{0}-\alpha\right)\left(1+2 \lambda+\beta+\alpha x_{0}+\alpha \lambda x_{0}+2 \alpha x_{0}^{2}-\alpha-\lambda \alpha\right)-\left[\beta+\lambda+\alpha \lambda\left(2 x_{0}^{2}+x_{0}-1\right)\right]>
\end{gathered}
$$

The characteristic equation of system (2) evaluated at the equilibrium point $E_{2}$ is:

$$
\begin{aligned}
\Lambda^{3}+(2 & \left.+\lambda-\alpha-\alpha x_{0}\right) \Lambda^{2}+\left(1+2 \lambda+\beta-\alpha x_{0}-\alpha \lambda x_{0}+2 \alpha x_{0}^{2}-\alpha-\lambda \alpha\right) \Lambda+\beta+\lambda \\
& +\alpha \lambda\left(2 x_{0}^{2}-x_{0}-1\right)=0 .
\end{aligned}
$$

Based on the Routh-Hurwitz conditions, Eq. (7) has all roots with negative real parts if and only if

$$
\begin{gathered}
2+\lambda-\alpha-\alpha x_{0}>0 \beta+\lambda+\alpha \lambda\left(2 x_{0}^{2}-x_{0}-1\right)>0 \\
\left(2+\lambda-\alpha-\alpha x_{0}\right)\left(1+2 \lambda+\beta-\alpha x_{0}-\alpha \lambda x_{0}+2 \alpha x_{0}^{2}-\alpha-\lambda \alpha\right)-\left[\beta+\lambda+\alpha \lambda\left(2 x_{0}^{2}-x_{0}-1\right)\right]>0
\end{gathered}
$$

By varying the parameter $\lambda$ from 0.005 to ${ }^{5}$ and the parameter $\alpha$ from ${ }^{0.1}$ to 100 , the stability boundaries of the equilibrium points are plotted in Fig. 1 for specific values of the parameter $\beta$.
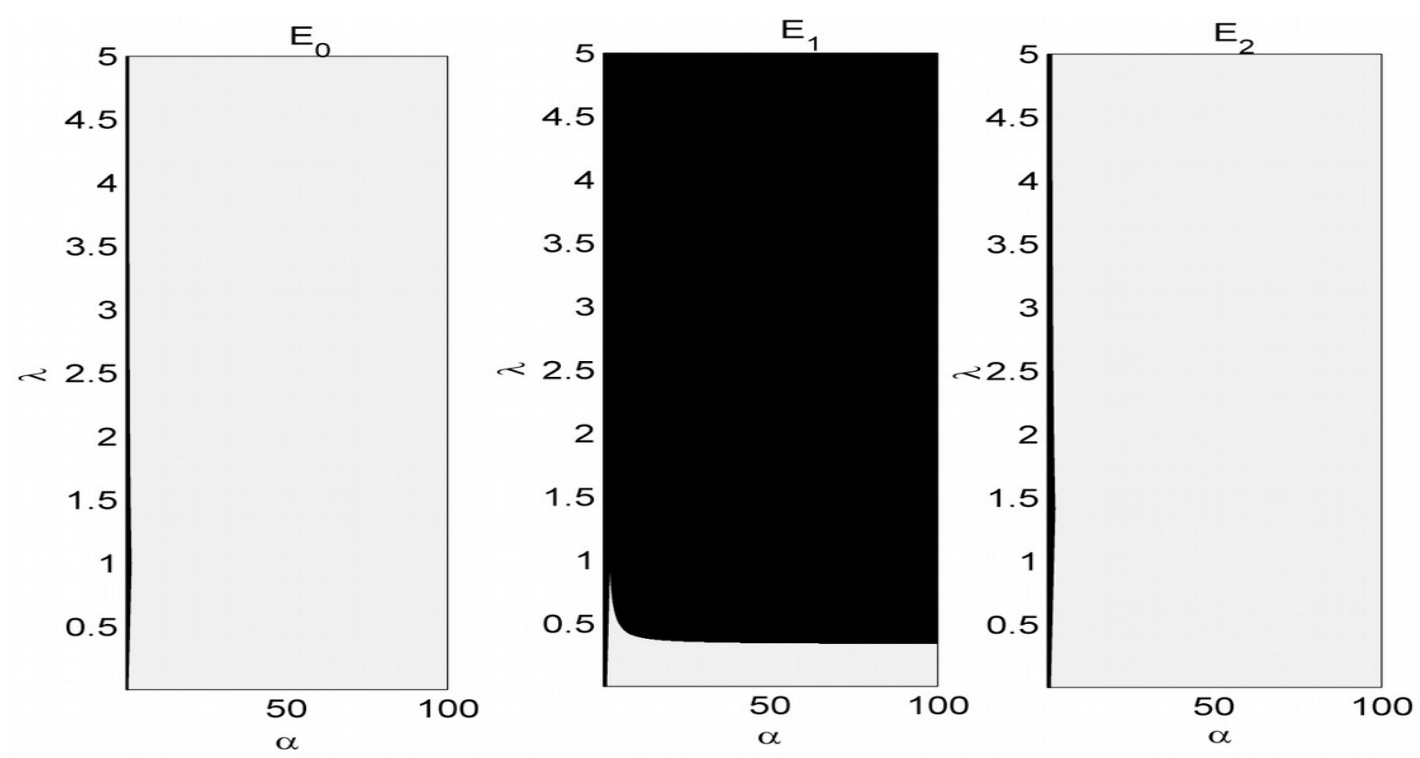

Fig. 1: (Color online) Stability diagram of the three equilibrium points with respect to versus the two parameters $\lambda$ and ${ }^{\alpha}$ for $\beta=1.01$. 
In Fig. 1, the black areas indicate the regions where equilibrium points are asymptotically stable while gray region represents the area where the equilibrium points are unstable. Since the equilibrium points change stability properties for specific parameters values of $\alpha$ and $\lambda$, system (2) has either a Hopf or transcritical bifurcation at those specific parameters values of $\alpha$ and ${ }^{\lambda}$.

The dynamical behaviours of the system (2) are described in terms of bifurcation diagrams, Lyapunov exponents, phase portraits and cross section of the basin of attraction. The parameters $\alpha, \lambda$ are fixed to $\alpha=20, \lambda=1.2$ and the bifurcation diagrams depicting the local extrema of $x(t)$ and the largest Lyapunov exponent (LLE) versus the parameter $\beta$ are plotted in Fig. 2. When the parameter varies from 1 to 3 (see Fig. 2 (a)), the trajectories of system (2) converge to one of the steady states. For, the system (2) exhibits chaotic behavior behavior followed at by an abrupt transition to period-1-oscillations. When performing the same analysis by ramping the parameter (see Fig. 2 (b)), the trajectories of system (2) converge to one of the steady states. For, the system (2) presents chaotic behavior followed at by an abrupt transition to period-1-oscillations. By comparing Figs. 2 (a) and (b), one can notice that the system (2) displays the coexistence of attractors between chaotic and steady state behaviors in the range. While in the range, the system (2) displays the coexistence of attractors between chaotic and periodic behaviors. The LLE shown in Fig. 2 (c) confirms the results found in Figs. 2 (a) and (b). The chaotic behaviour found in Fig. 2 is presented in Fig. 3 which shows the phase portrait of system (2) for a specific value of the parameter. 


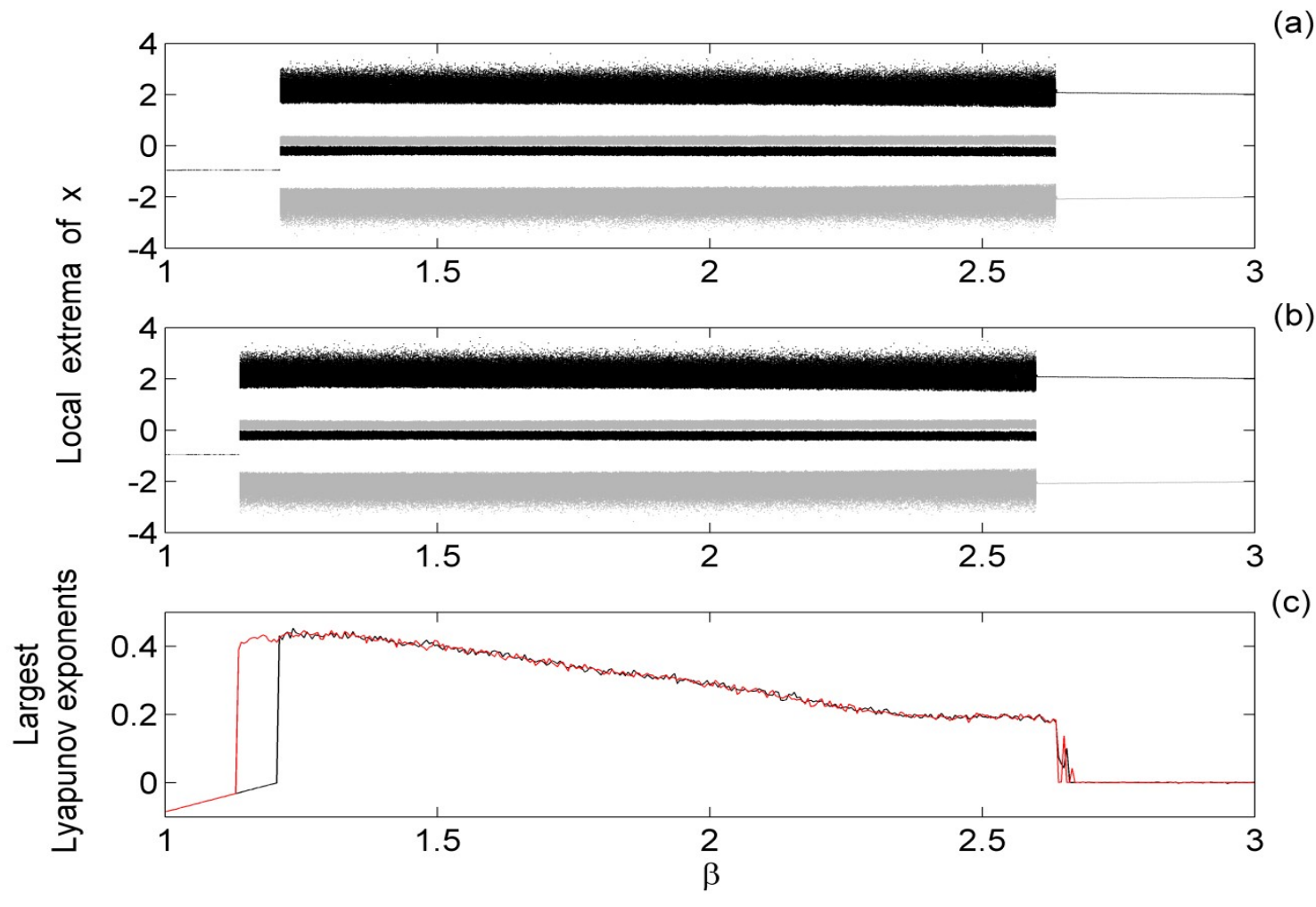

Fig. 2: (Color online) The bifurcation diagram depicting the local maxima (black dots) and local minima (gray dots) of $x^{x}(t)$ (a) and (b) and the LLE (c) versus the parameter $\beta$ for $\alpha=20$ and $\lambda=1.2$. Bifurcation diagrams are obtained by scanning the parameter $\beta$ upwards (a) and downwards (b). LLE are obtained by scanning the parameter $\beta$ upwards (black) and downwards (red).
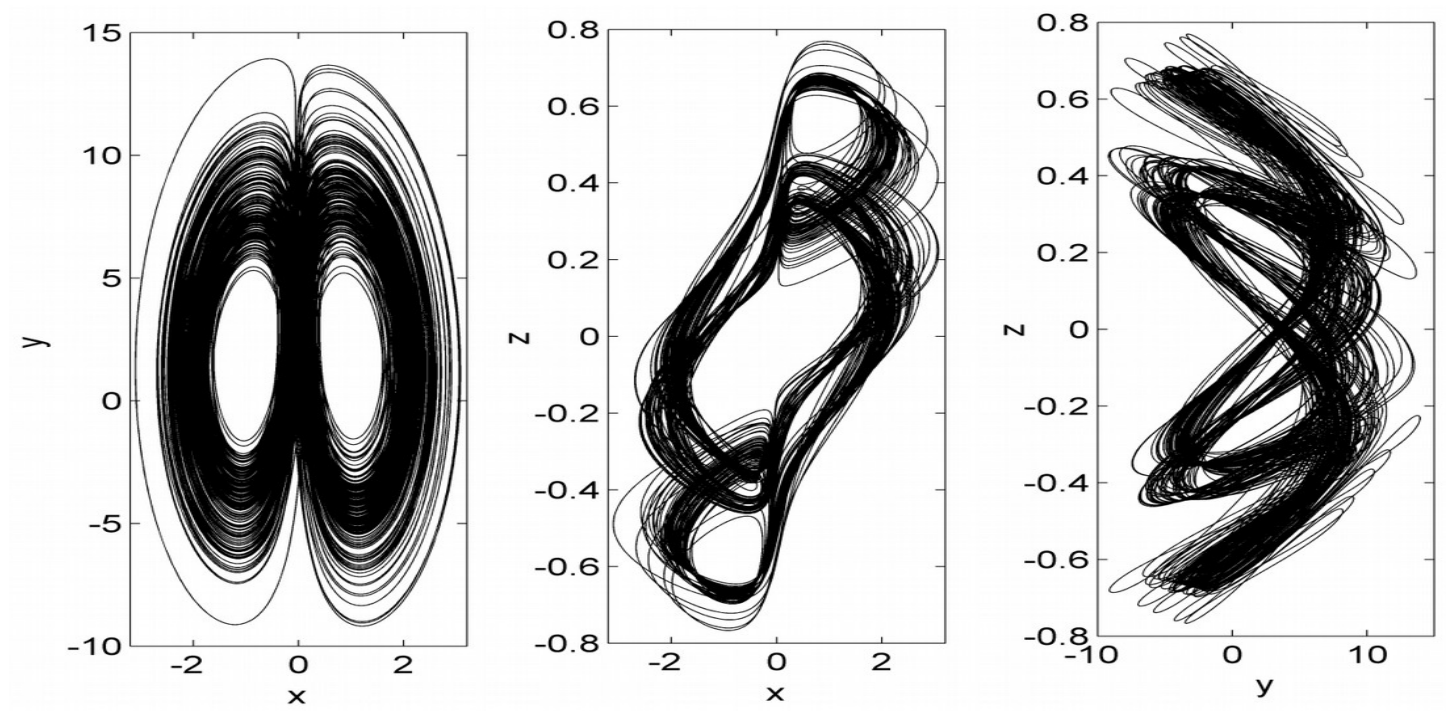

Fig. 3: The phase portraits of system (2) in planes $(x, y),(x, z)$ and $(y, z)$ for $\beta=2, \alpha=20$ and $\lambda=1.2$. The initial conditions are $(x(0), y(0), z(0))=(-1.5,-1.5,0.75)$. 

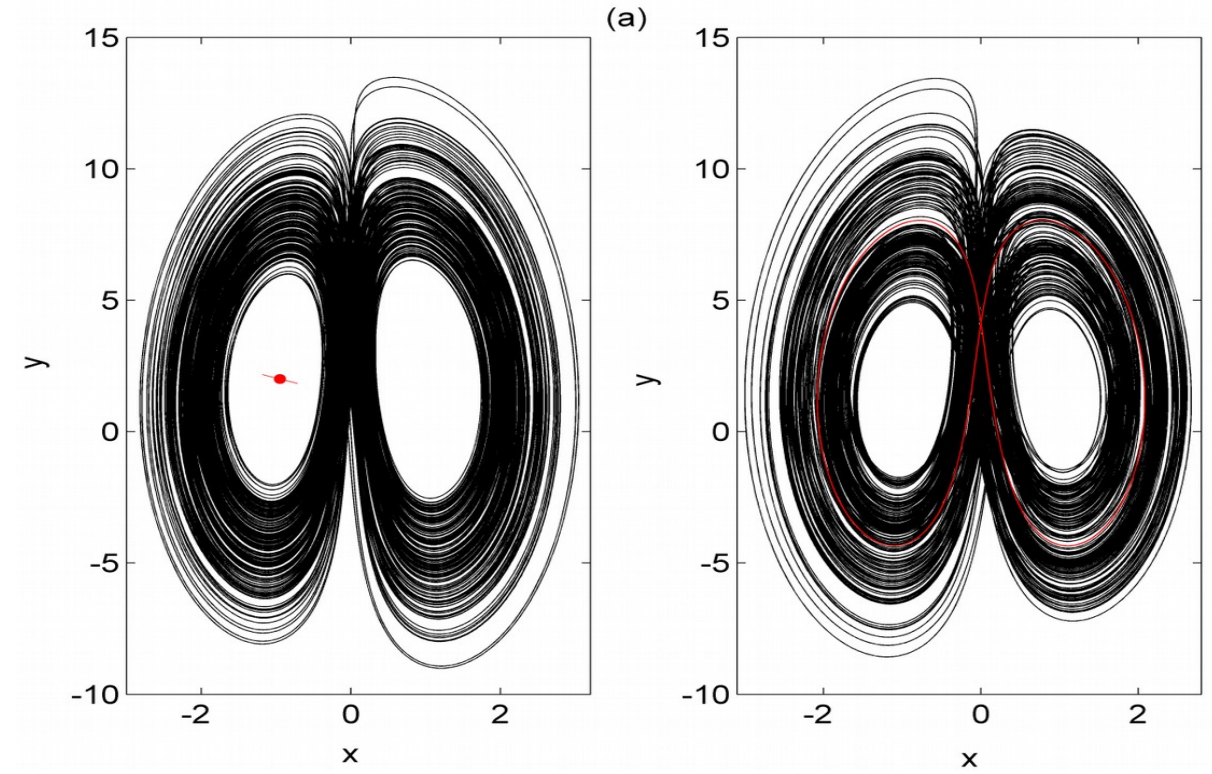

(b)

Fig. 4: (Color online) Coexisting attractors for specific values of $\beta$ and initial conditions: (a) $\beta=1.2$ and (b) $\beta=2.62$. The curves in black color are obtained using the initial conditions are $(x(0), y(0), z(0))=(-1.5,-$ $1.5,0.75)$ while the curves in red color are obtained using the initial conditions $(x(0), y(0), z(0))=(-0.9,-1.5$, $0.75)$ in panel (a) and $(x(0), y(0), z(0))=(-0.33,-1.5,0.75)$ in panel (b). The remaining parameters are given in Fig. 2.

The system (2) exhibits double-scroll chaotic attractor as shown in Fig. 3. The coexisting attractors is further detailed in Fig. 4 which depicts the phase portraits of the resulting attractors of the system (2) in the plane ${ }^{(x, y)}$ for specific value of the parameter $\beta$ and initial conditions.

At $\beta=1.2$, the system (2) displays steady state and chaotic behaviours for the two different initial conditions as shown in Figs. 4 (a). Periodic and chaotic behaviours are revealed in Fig. 4 (b) at ${ }^{\beta=2.62}$ for two different initial conditions. The basin of attraction of system (2) in the plane ${ }^{z=0.75}$ is presented in Fig. 5. In Fig. 5, the system (2) exhibits either chaotic or periodic behaviors depending to the initial conditions.

In Figure 6, the bifurcation diagram depicting the local extrema of $x(t)$ and the LLE versus the parameter $\alpha$ are plotted for $\beta=1.01$ and $\lambda=1$. In Figure 6 (a) when the parameter $\alpha$ varies from 0 to 100 , the trajectories of system (2) converge to the steady state point $E_{0}=(0, \alpha, 0)$ up to $\alpha \approx 2$ where Hopf bifurcation occurs followed by a period-doubling route to chaotic behaviour. The dynamical behaviour found in Fig. 6 (a) is confirmed by the LLE shown in Fig. 6 (b). The chaotic behaviour found in Fig. 6 is presented in Fig. 7 which shows 
the phase portrait of system (2) for a specific value of the parameter ${ }^{\alpha}$. The system (2) exhibits double-scroll chaotic attractor as shown in Fig. 7. The shape of double-scroll chaotic attractor of Fig. 7 is different to those of Fig. 3.

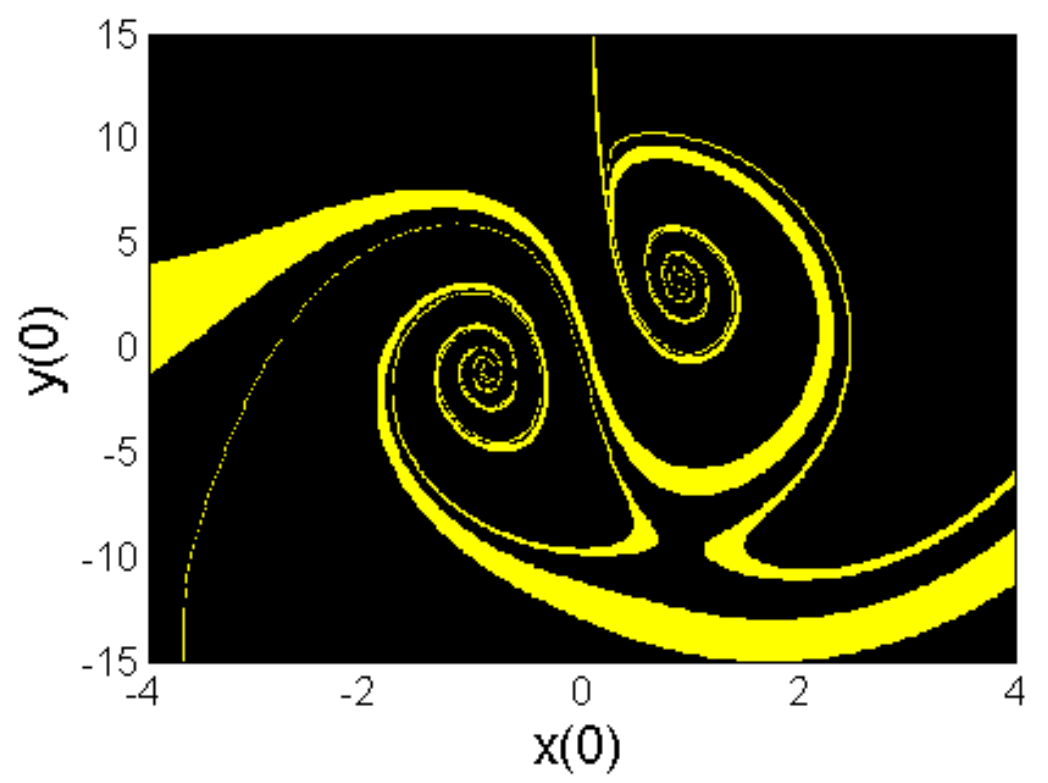

Fig. 5: (Color online) Cross section of the basin of attraction of system (2) in the plane ${ }^{z=0.75}$ for $\beta=2.62, \alpha=20$ and $\lambda=1.2$. Initial conditions in black and yellow regions lead respectively to chaotic and periodic behaviors.

(a)

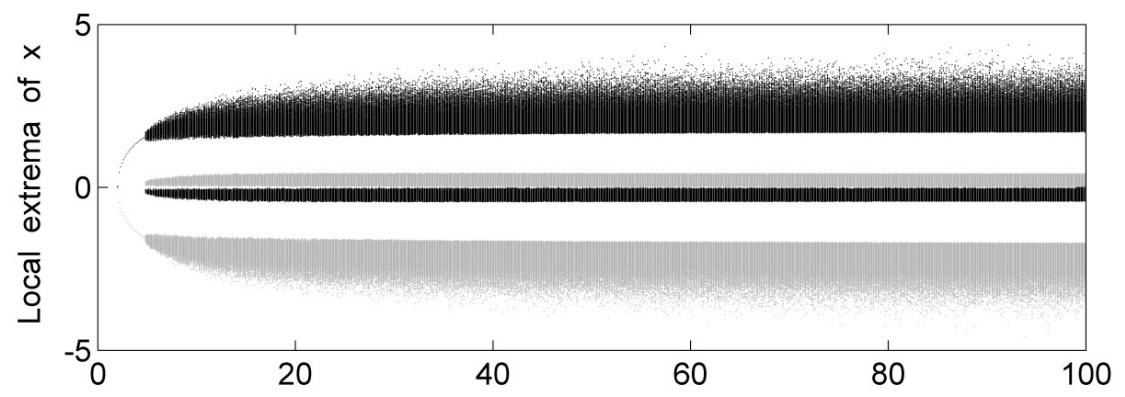

(b)

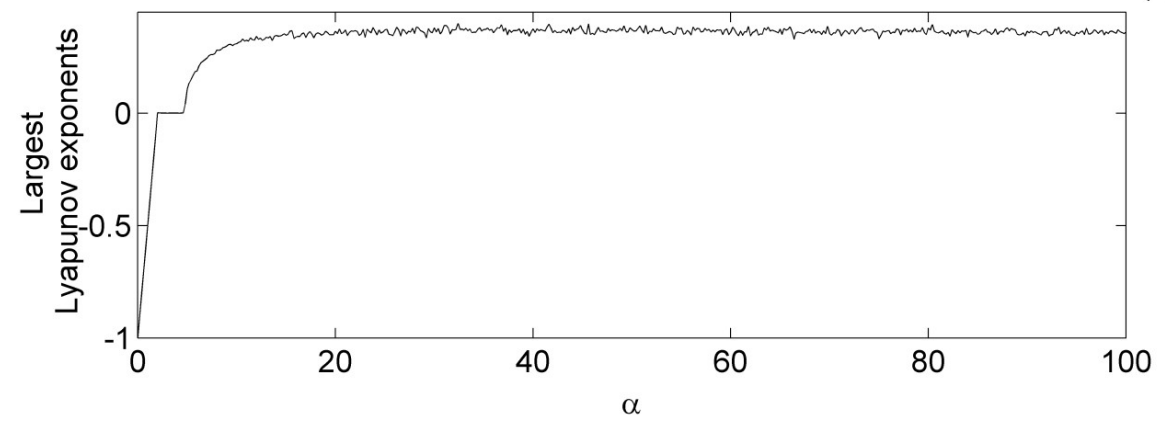

Fig. 6: Bifurcation diagram depicting the local maxima (black dots) and local minima (gray dots) of $x(t)$ (a) and LLE (b) versus parameter ${ }^{\alpha}$ for $\beta=1.01$ and $\lambda=1$. 

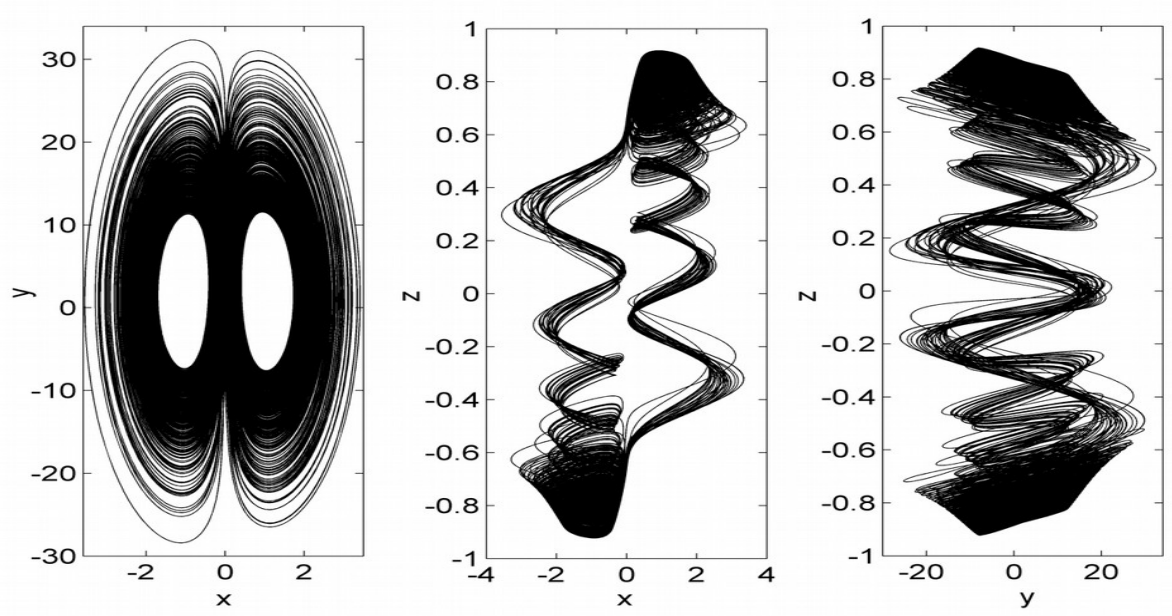

Fig. 7: The phase portraits of system (2) in planes $(x, y),(x, z)$ and $(y, z)$ for $\alpha=100, \beta=1.01$ and $\lambda=1$. The initial conditions are $(x(0), y(0), z(0))=(-1.5,-1.5,0.75)$.

\section{Chaos control of $3 D$ chaotic system using single controller}

A single state feedback controller is used in this section to stabilize the chaotic behavior found in the $3 \mathrm{D}$ chaotic system. Therefore, two single controllers are mathematically designed by using the principle of Lyapunov's method for asymptotic global stability [32].

\subsection{Proposed controller 1}

In this subsection, the controller $u_{1}$ is added to the equation (1a):

$$
\begin{aligned}
& \frac{d x}{d t}=x(y-1)-\beta z+u_{1}, \\
& \frac{d y}{d t}=\alpha\left(1-x^{2}\right)-y, \\
& \frac{d z}{d t}=x-\lambda z,
\end{aligned}
$$

where $u_{1}=\beta z-x y$. Substituting the expression of the controller ${ }^{u_{1}}$ into the controlled system (9) becomes

$$
\begin{aligned}
& \frac{d x}{d t}=-x, \\
& \frac{d y}{d t}=\alpha\left(1-x^{2}\right)-y,
\end{aligned}
$$




$$
\frac{d z}{d t}=x-\lambda z
$$

The solution of Eq. (9a) is $x(t)=x(0) e^{-t}$. That is yield $\lim _{t \rightarrow \infty} x(t)=0$. Thus, the system (10) can be reduced as follows:

$$
\begin{aligned}
& \frac{d y}{d t}=\alpha-y, \\
& \frac{d z}{d t}=-\lambda z,
\end{aligned}
$$

The solution of system (11) can be rewritten as follows:

$$
y(t)=\alpha+y(0) e^{-t}, z(t)=z(0) e^{-\lambda t} .
$$

That is yield $\lim _{t \rightarrow \infty} y(t)=\alpha$ and $\lim _{t \rightarrow \infty} z(t)=0$. Therefore, the following theorem is given:

Theorem 1: The chaotic behavior found in the 3D chaotic system can be controlled using the controller $u_{1}=\beta z-x y$.

Proof: The proof is obvious, so leave it out.

The curves of the state responses and the output of the controller are shown in Fig. 12. In Fig.

12, the controller $u_{1}$ is activated at $t \geq 1400$. It is noted that Theorem 1 is effective.

\subsection{Proposed controller 2}

In this subsection, the controller $u_{2}$ is added to the equation (2b):

$$
\begin{aligned}
& \frac{d x}{d t}=x(y-1)-\beta z, \\
& \frac{d y}{d t}=\alpha\left(1-x^{2}\right)-y+u_{2}, \\
& \frac{d z}{d t}=x-\lambda z,
\end{aligned}
$$

where $u_{2}=\alpha\left(x^{2}-1\right)$. Substituting the expression of the controller $u_{2}$ into the controlled system (12) becomes

$$
\begin{aligned}
& \frac{d x}{d t}=x(y-1)-\beta z, \\
& \frac{d y}{d t}=-y,
\end{aligned}
$$




$$
\frac{d z}{d t}=x-\lambda z
$$

The solution of Eq. (14b) is $y(t)=y(0) e^{-t}$. That is yield $\lim _{t \rightarrow \infty} y(t)=0$. Thus, the system (14) can be reduced as follows:

$$
\left(\begin{array}{l}
\frac{d x}{d t} \\
\frac{d z}{d t}
\end{array}\right)=\left(\begin{array}{cc}
-1 & -\beta \\
1 & -\lambda
\end{array}\right)\left(\begin{array}{l}
x \\
z
\end{array}\right)=A\left(\begin{array}{l}
x \\
z
\end{array}\right)
$$

For $\alpha=20, \beta=2$ and $\lambda=1.2$, the eigenvalues of $\mathrm{A}$ at the equilibrium point $(x=0, y=0)$ are $\Lambda_{1,2}=-1.1 \pm 1.41067359 j$ with $j^{2}=-1$. Based on the Routh-Hurwitz criteria, the subsystem (15) is asymptotically stable. Then the following theorem is given:

Theorem 2: The chaotic behavior found in the 3D chaotic system can be controlled using the controller $u_{2}=\alpha\left(x^{2}-1\right)$.

Proof: The proof is obvious, so leave it out.
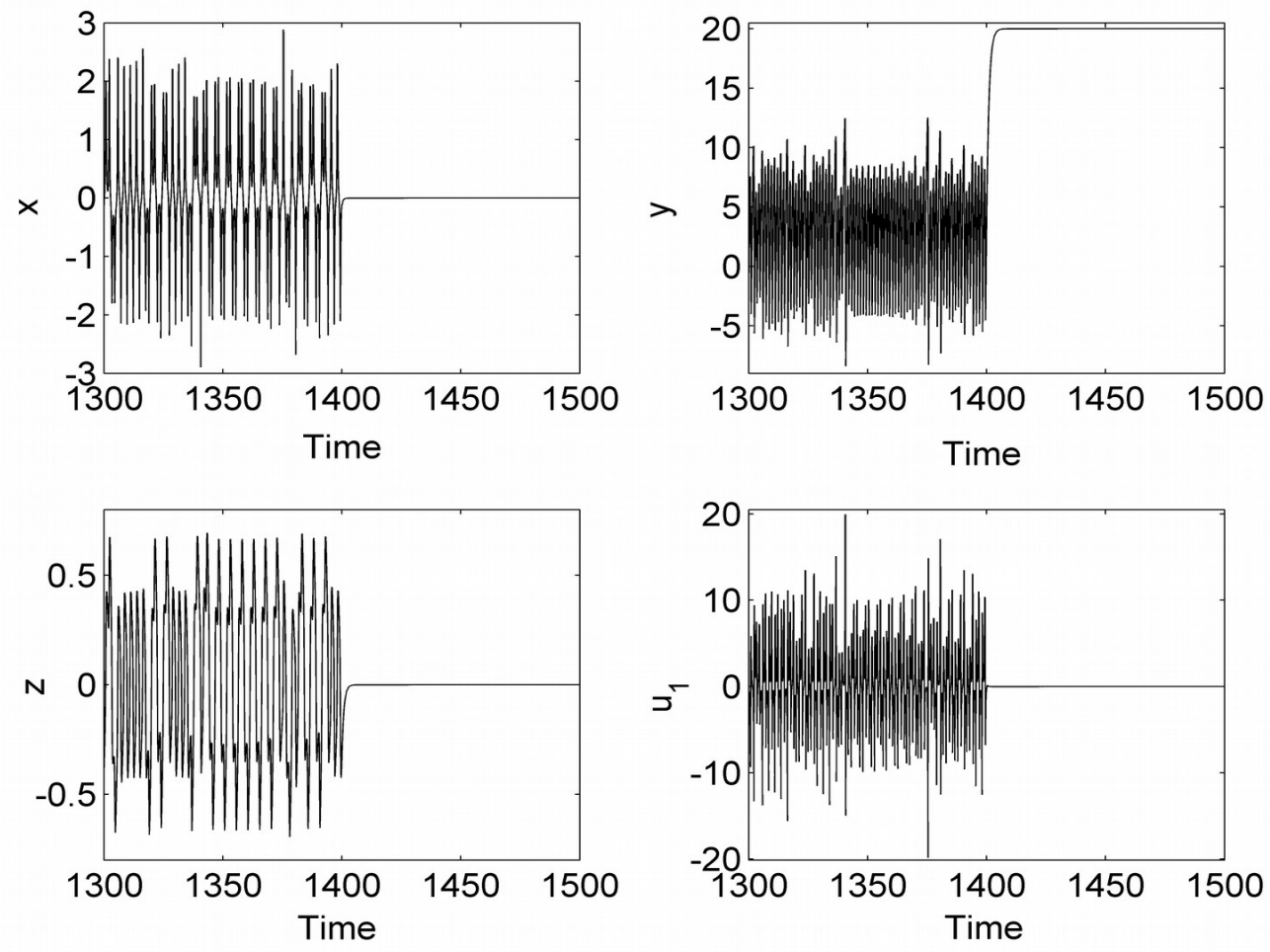

Fig. 12 Time series of $x, y, z$ and the output of the controller $u_{1}$ for $\alpha=20, \beta=2$ and $\lambda=1.2$. The initial conditions are $(x(0), y(0), z(0))=(-1.5,-1.5,0.75)$. 

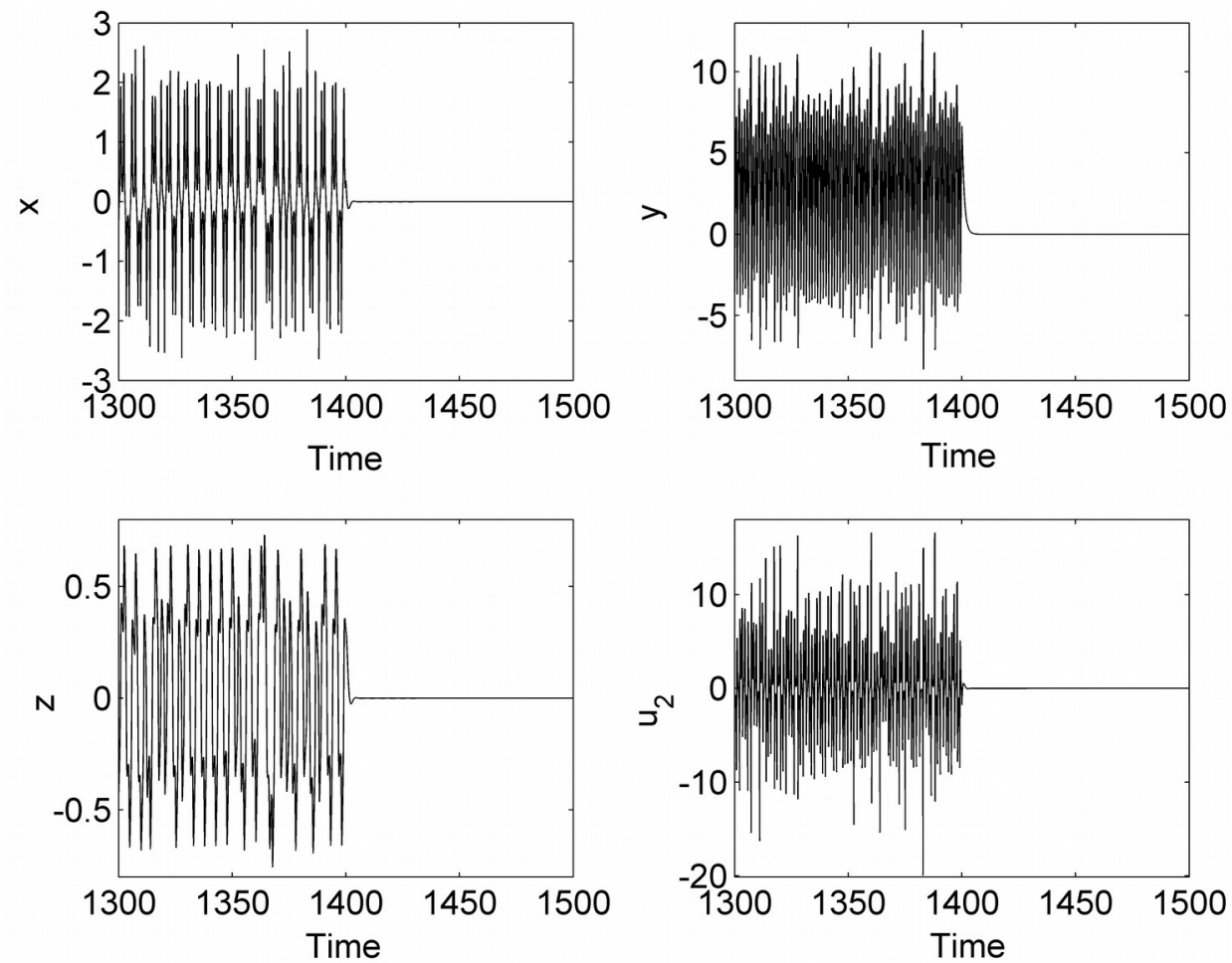

Fig. 13 Time series of $x, y, z$ and the output of the controller $u_{2}$ for $\alpha=20, \beta=2$ and $\lambda=1.2$. The initial conditions are $(x(0), y(0), z(0))=(-1.5,-1.5,0.75)$.

The curves of the state responses and the output of the controller are shown in Fig. 13. In

Fig. 13, the controller $u_{2}$ is activated at $t \geq 1400$. It is noted that Theorem 2 is effective. It is interesting to point out here that from practical realization point of view, the single controller 2 is preferred because of the inclusion of one state variable (i.e. x) signifying a lesser requirement of sensing devices during their fabrication. Hence, this making the system to become cheap.

\section{Chaos synchronization of unidirectional coupled identical 3D chaotic systems}

In this section, a single feedback control method is used to synchronize unidirectional coupled identical 3D chaotic systems. The drive and the response 3D chaotic systems are expressed respectively:

$$
\frac{d x_{1}}{d t}=x_{1}\left(y_{1}-1\right)-\beta z_{1}
$$




$$
\begin{aligned}
& \frac{d y_{1}}{d t}=\alpha\left(1-x_{1}^{2}\right)-y_{1}, \\
& \frac{d z_{1}}{d t}=x_{1}-\lambda z_{1}, \\
& \frac{d x_{2}}{d t}=x_{2}\left(y_{2}-1\right)-\beta z_{2}+u_{3}, \\
& \frac{d y_{2}}{d t}=\alpha\left(1-x_{2}^{2}\right)-y_{2}, \\
& \frac{d z_{2}}{d t}=\sigma\left(y_{2}-z_{2}\right),
\end{aligned}
$$

where ${ }^{u_{3}}$ are controllers to achieve synchronization between the systems (16) and (17). The synchronization errors are defined as $e_{1}=x_{2}-x_{1}, e_{2}=y_{2}-y_{1}, e_{3}=z_{2}-z_{1}$. Its derivatives are given below:

$$
\begin{aligned}
& \frac{d e_{1}}{d t}=-e_{1}-\beta e_{3}+x_{2} e_{2}+y_{1} e_{1}+u_{3}, \\
& \frac{d e_{2}}{d t}=-e_{2}-e_{1}\left(x_{1}+x_{2}\right), \\
& \frac{d e_{3}}{d t}=e_{1}-\lambda e_{3},
\end{aligned}
$$

where the controllers are chosen as $u_{3}=\beta e_{3}-x_{2} e_{2}-y_{1} e_{1}$. Substituting the expressions of the controllers $u_{3}$ into the system (18) becomes

$$
\begin{aligned}
& \frac{d e_{1}}{d t}=-e_{1}, \\
& \frac{d e_{2}}{d t}=-e_{2}-e_{1}\left(x_{1}+x_{2}\right), \\
& \frac{d e_{3}}{d t}=e_{1}-\lambda e_{3} .
\end{aligned}
$$

The solution of the Eq. (18a) is $e_{1}(t)=e_{1}(0) e^{-t}$. That is yield $\lim _{t \rightarrow \infty} e_{1}(t)=0$ and $\lim _{t \rightarrow \infty} e_{2}(t)=0$. Thus, the system (19) can be reduced as follows:

$$
\frac{d e_{2}}{d t}=-e_{2}
$$




$$
\frac{d e_{3}}{d t}=-\lambda e_{3}
$$

The solutions of the Eqs. (19a) and (19b) are $e_{2}(t)=e_{2}(0) e^{-t}$ and $e_{3}(t)=e_{3}(0) e^{-\lambda t}$, respectively. That is yield $\lim _{t \rightarrow \infty} e_{2}(t)=0$ and $\lim _{t \rightarrow \infty} e_{3}(t)=0$. Therefore, the following theorem is given:

Theorem 3: The controllers $u_{3}=\beta e_{3}-x_{2} e_{2}-y_{1} e_{1}$ can synchronize the drive system (16) and the response system (16).

Proof: The proof is obvious, so leave it out.

For numerical simulations, the initial conditions of the drive system (16) and the response system (16) are

$$
\left(x_{1}(0), y_{1}(0), z_{1}(0)\right)=(-1.5,-1.5,0.75) \text { an }\left(x_{2}(0), y_{2}(0), z_{2}(0)\right)=(1.5,-1.5,-0.75) \text {, }
$$

respectively. The dynamics of the synchronization errors and the output of the controller $u_{3}$ for $\alpha=20, \beta=2$ and $\lambda=1.2$ is depicted in Fig. 14. In Fig. 14, the controller $u_{3}$ is activated at $t \geq 1400$. It is noted that Theorem 3 is effective. 

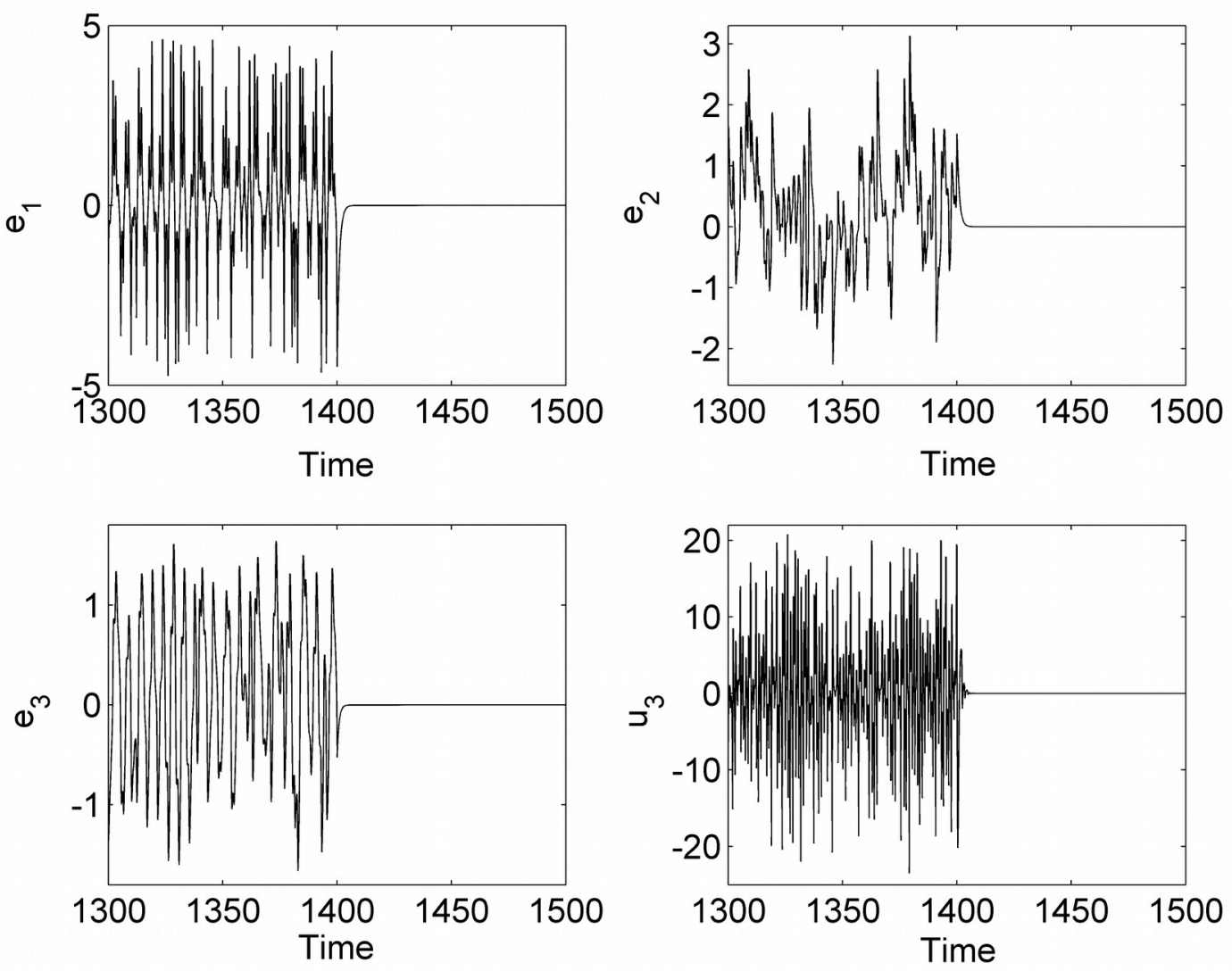

Fig. 14 Time series of synchronization error variables and the output of the controller $u_{3}$ for $\alpha=20$, $\beta=2$ and $\lambda=1.2$.

\section{Conclusion}

This paper deals with the analysis, chaos control and synchronization of a proposed threedimensional autonomous system. It was found during the numerical simulations that the proposed three-dimensional autonomous system exhibits double-scroll chaotic attractors and coexisting attractors emerged. Moreover, the two proposed single controllers were designed to suppress the chaotic behavior in proposed three dimensional autonomous. Among these two controllers, the single controller 2 could be a preferable choice because of its use of one state variable (i.e. x). Finally, the synchronization of unidirectional coupled identical proposed three dimensional autonomous was achieved using a single controller.

\section{References}

[1] Tlelo-Cuautle, E., Pano-Azucena, A. D., Rangel-Magdaleno, J. J., Carbajal-Gomez, V. H., and Rodriguez-Gomez, G. (2016), "Generating a 50-scroll chaotic attractor at 66 MHz by using FPGAs”, Nonlinear Dynamics, Vol.85, No 4, pp. 2143-2157. 
[2] Rajagopal, K., Karthikeyan, A., and Duraisamy, P. (2017), "Hyperchaotic Chameleon: Fractional order FPGA implementation”. Complexity, Vol. 2017, pp.8979408.

[3] Li, C. B., Sprott, J. C., Kapitaniak, T. and Lu, T. A. (2018), "Infinite lattice of hyperchaotic strange attractors", Chaos Solit. Fract. Vol.109, pp.76-82.

[4] Akgul, A., Kacar, S., Pehlivan I., and Aricioglu, B. (2018), "Chaos-based encryption of multimedia data and design of security analysis interface as an educational tool". Comput. Appl. Eng. Educ., Vol.26, pp.1336-1349.

[5] Arshad U, Khan, M., Shaukat, S., Amin, M., and Shah, T. (2019), “An Efficient Image Privacy Scheme Based on Nonlinear Chaotic System and Linear Canonical Transformation", Physica A: Statistical Mechanics and its Applications, https://doi.org/10.1016/j.physa.2019.123458.

[6] Hilbert, D. (1901), "Mathematical problems". Bull. Amer. Math. Soc. Vol. 8, pp. 437479.

[7] Blekhman, I., Indeitsev, D. A., and Fradkov, A.L. (2007), "Slow motions in systems with inertially excited vibrations”. IFAC Proceedings Vol. 40, No.14, pp. 126-131.

[8] Hide, R., Skeldon, A. C., and Acheson, D. J. (1996), "A study of two novel self-exciting single-disk homopolar dynamos: Theory”, Proc. R. Soc. Lond. A, Vol. 452, pp.13691395.

[9] Moroz, M., Hide, R., and Soward, A. M. (1998), “On self-exciting coupled Faraday disk homopolar dynamos driving series motors", Physica D, Vol.117, pp. 128-144.

[10]Knobloch, E. (1981), "Chaos in the segmented disc dynamo”, Phys. Lett. A, Vol. 82, pp. 439-440.

[11]Wei, Z.C., Akgul A., Kocamaz, U. E., Moroz, I., and Zhang, W. (2018), "Control, electronic circuit application and fractional-order analysis of hidden chaotic attractors in the self-exciting homopolar disc dynamo", Chaos, Solitons and Fractals, Vol. 111, pp.157-168.

[12]Liu, Y.B., Li, J.Z., Wei, Z.C. and Moroz, I. (2018), "Bifurcation analysis and integrability in the segmented disc dynamo with mechanical friction". Advances in Difference Equations, Vol. 2018, pp. 210.

[13]Leonov, G.A., Kuznetsov, N.V., and Vagaitsev, V.I. (2011), "Localization of hidden Chua's attractors", Phys. Lett. A, Vol. 375, pp. 2230-2233.

[14] G.A. Leonov , N.V. Kuznetsov, Hidden attractors in dynamical systems: from hidden oscillations in Hilbert-Kolmogorov, Aizerman, and Kalman problems to hidden chaotic attractor in Chua circuits, Int. J. Bifurc. Chaos 23 (2013) 1330002.

[15]G.A. Leonov, Kuznetsov, N.V. , Vagaitsev, V.I. (2012) "Hidden attractor in smooth Chua systems, Physica D 241 1482-1486.

[16]Dudkowski, D., Prasad A., and Kapitaniak T. (2015) "Perpetual points and hidden attractors in dynamical systems", Phys. Lett. A, 379, pp. 2591-2596.

[17]Dudkowski, D., Prasad A., and Kapitaniak T. (2018), "Describing chaotic attractors: regular and perpetual points", Chaos, Vol. 28, pp. 033604.

[18]Perc, M. (2005) "Visualizing the attraction of strange attractors", Eur. J. Phys., Vol. 26, $579-587$. 
[19] Silva, P.H.O., Nardo, L.G., Martins, S.A.M., Nepomuceno, E.G., and Perc, M. (2018), "Graphical interface as a teaching aid for nonlinear dynamical systems", Eur. J. Phys., Vol. 39, pp. 065105.

[20]Liu Y.J., Yang Q.G. (2010), "Dynamics of a new Lorenz-like chaotic system”, Nonlinear Anal. Real World Appl., Vol. 11, pp. 2563-2572.

[21]Li, C.B., Sprott, J.C. (2018), “An infinite 3-D quasiperiodic lattice of chaotic attractors", Phys. Lett. A, Vol. 382, pp. 581-587.

[22]Li, C.B. , Sprott, J.C., Kapitaniak T., and Lu T.A. (2018), "Infinite lattice of hyperchaotic strange attractors", Chaos Solitons Fractals, Vol.109, pp. 76-82.

[23]Jafari, S., Sprott, J. C., and Golpayegani, S. (2013) "Elementary quadratic chaotic flows with no equilibria", Phys. Lett. A, Vol. 377, pp. 699-702.

[24]Jafari, M. A., Mliki, E., Akgul, A., Pham, V. T., Kingni, S. T., Wang, X. and Jafari, S. (2017), "Chameleon: The most hidden chaotic flow", Nonlin. Dyn., Vol. 88, pp. 23032317.

[25]Wei, Z.C., Zhang, W., Wang, Z., and Yao, M.H. (2015), "Hidden attractors and dynamical behaviors in an extended Rikitake system", Int. J. Bifurc. Chaos, Vol. 25, pp. 1550028 .

[26] Muthukumar, P., Balasubramaniam, P., and Ratnavelu, K. (2015), "Detecting chaos in a system of four disk dynamos and its control”, Nonlinear Dyn., Vol. 83, pp. 2419-2426.

[27] Wei Z.C., Moroz, I., Sprott, J.C., Akgul, A., and Zhang W. (2017), "Hidden hyperchaos and electronic circuit application in a 5D self-exciting homopolar disc dynamo", Chaos, Vol. 27, pp. 033101.

[28]Wei Z.C., Moroz, I., Sprott, J.C., Wang, Z., and Zhang W. (2017), "Detecting hidden chaotic regions and complex dynamics in the self-exciting homopolar disc dynamo", Int. J. Bifurc. Chaos, Vol. 27, pp. 1730008.

[29]Feng, Y., Pan, W.Q. (2017), "Hidden attractors without equilibrium and adaptive reduced-order function projective synchronization from hyperchaotic Rikitake system", Pramana J. Phys., Vol. 88, pp. 62.

[30]Bao, J.H., Chen, D.D. (2017), “Coexisting hidden attractors in a 4D segmented disc dynamo with one stable equilibrium or a line equilibrium", Chin. Phys. B, Vol. 26, pp. 1056-1674.

[31]Wei, Z.C., Zhu, B., Yang, J., Perc, M., and Slavinec, M. (2019), "Bifurcation analysis of two disc dynamos with viscous friction and multiple time delays". Applied Mathematics and Computation, Vol. 347, pp. 265-281.

[32]He, P., Li, Y.M. (2015), "Control and synchronization of a hyperchaotic finance system via single controller scheme". International Journal of Intelligent Computing and Cybernetics, Vol.8, No. 4, pp. 330-344. 